Article

\title{
A stochastic binary model for regulation of gene expression to investigate treatment effects targeting RKIP
}

\author{
Guilherme Giovanini ${ }^{1}$, Luciana Rodrigues Carvalho Barros ${ }^{2}{ }^{(}$, Leonardo dos Reis Gama ${ }^{2}$, Tharcisio Citrangulo \\ Tortelli Junior ${ }^{2}$, Alexandre Ferreira Ramos ${ }^{1,2, *}$ (D) \\ 1 Escola de Artes, Ciências e Humanidades, Universidade de São Paulo, Av. Arlindo Béttio, 1000, 03828-000, \\ São Paulo, SP, Brazil; \\ 2 Centro de Investigação Translacional em Oncologia, Departamento de Radiologia e Oncologia, Faculdade de \\ Medicina da Universidade de São Paulo \& Instituto do Câncer do Estado de São Paulo, Av. Dr. Arnaldo, 251, \\ 01246-000, São Paulo, SP, Brazil. \\ * Correspondence: alex.ramos@usp.br
}

\begin{abstract}
In this manuscript we use an exactly solvable stochastic binary model for regulation of gene expression to analyse the dynamics of response to a treatment aiming to modulate the number of transcripts of RKIP gene. We demonstrate the usefulness of our method simulating three treatment scenarios aiming to reestablish RKIP gene expression dynamics towards pre-cancerous state: $i$. to increase the promoter's ON state duration; ii. to increase the mRNAs' synthesis rate; iii. to increase both rates. We show that the pre-treatment kinetic rates of ON and OFF promoter switching speeds and mRNA synthesis and degradation will affect the heterogeneity and time for treatment response. Hence, we present a strategy for reducing drug dosage by simultaneously targeting multiple kinetic rates. That enables a reduction of treatment response time and heterogeneity which in principle diminishes the chances of emergence of resistance to treatment. This approach may be useful for inferring kinetic constants related to expression of antimetastatic genes or oncogenes and on the design of multi-drug therapeutic strategies targeting master regulatory genes.
\end{abstract}

Keywords: RKIP expression regulation; Stochastic binary regulation of gene expression; Treatment targeting RKIP levels increase; Reduction of heterogeneity of treatment response

\section{Introduction}

Despite recent advances in our understanding of cancer biology, the use of quantitative methods to integrate the plethora of generated data to design treatments targeting metastasis is still in its infancy [1,2]. The genotypic variability and intrinsic randomness of biochemical reactions governing epigenetics underpin the multiplicity of cancer cell phenotypes commonly termed as tumor heterogeneity [3-8]. Additionally, cellular processes are controlled by several networks of chemical reactions characterized by changeable topologies and functional redundancies that equip cells with adaptation and robustness capabilities [9-12]. And, the numerous characteristic timescales of the chemical processes taking place inside the cell adds one more layer of cumbersomeness [13,14]. Thus, the enhancement of therapeutic strategies requires the analysis and the "engineering" of the dynamics of treatment response in a complex system composed by several interacting components having a multiplicity of characteristic time scales and subjected to randomness. Finding building blocks having those features may provide useful insights on how to orchestrate the dynamics of a such a complex system [15]. For that, the exactly solvable stochastic model for transcription of a binary gene [16-19] is a good candidate to be used as a prototype for simulating enhanced treatment strategies.

To develop our theoretical approach for therapeutic design we start selecting the gene encoding the Raf kinase inhibitory protein (RKIP) as a model system because it plays a major role on regulation of the dynamics of multiple components of a cell [20]. Because 
cancer lethality is mainly caused by metastasis, the choice of RKIP is promising as its concentrations are typically reduced in metastatic cancers [21-23]. That negative correlation turns RKIP and RKIP-related gene signatures into useful biomarkers of metastatic risk in cancer patients [24]. The characterization of RKIP as an anti-metastatic gene is reinforced by experiments demonstrating that its overexpression blocks in vivo invasion and metastatic progression $[25,26]$. Hence, we focus on possible strategies to increase the amounts of RKIP in cancer cells by re-modulating its expression profiles towards the pre-cancer regimens.

For that goal, we simulate the effect of application of multiple drugs each targeting a different kinetic rate of a two state stochastic model for gene transcription. Because of treatment, we will consider the kinetic rates of the model to be time-dependent in response to drug application. That enables one to estimate the speed and heterogeneity of response to treatment by, respectively, computing the dynamics of the average number of transcripts and their variance. The exact solutions of the model at constant kinetic rates have two characteristic time scales, one related with the promoter state switching and the second being the mRNA lifetime. Recently, we demonstrated that the ratio between those two time scales may be used to classify the qualitatively distinguishable noise regimens of gene transcription on the binary model $[19,27]$ and the reliability of information about the promoter state that is transmitted by gene products [28]. Here, we assume that the addition of a drug cause the kinetic rates of the model to become time dependent and that this effect decays exponentially such that has up to four additional time scales for the problem. Then, we show that treatment targeting a gene having a fast switching promoter, and expressing as a quasi-Poissonian process, enables the fastest and least heterogeneous response to treatment. If the gene has a slow switching promoter, the response will be slower and have higher heterogeneity. A gene expressing in bursty fashion will enable a fast response having maximal heterogeneity. Then, we build upon the previous analysis to design an enhanced treatment enabling a faster response with reduced heterogeneity that is independent of the pre-treatment time scales. The enhanced treatment is based on reduced drug dosages to reduce the chances of toxicity and emergence of resistance caused by compensatory effects $[1,2]$.

The remaining of this manuscript is organized as follows. The assumptions underlying the qualitative and quantitative models that we propose are presented on Section 2. The obtained results are shown on Section 3 and we discuss them on Section 4. Our concluding remarks are presented on Section 5.

\section{Methods and Models}

Gene expression is the key mechanism keeping cell function by means of an extensive molecular machinery which transforms the genetic information into molecular function. Gene expression can be described as a two stages process, namely, the gene transcription that generates the mRNA, and the mRNA translation which has proteins as their products. Here we focus on gene transcription and provide a simplified picture of how it happens in eukaryotic cells: the RNA Polymerase protein complex (RNAPolII) binds to a specific region of a gene, the promoter site, and begins transcription elongation. The binding of RNAPolII to the promoter site may be regulated by its interaction with a DNA region called enhancer. The enhancers are regions of the DNA that interact with the transcription factors that can provide a positive or negative regulation of the binding of RNAPolII to the promoter site. This is the process that we will use to model RKIP transcription.

\subsection{A brief description of the molecular role of RKIP}

RKIP protein is a regulator of kinases that directly binds to Raf kinase [29] and is involved on regulation of signalling pathways such as the Raf-Mek-Erk cascade and the NF- $\kappa$ B-related pathways $[30,31]$. Both pathways modulate cell proliferation, the former regulates differentiation, while the latter is responsible for inflammation, and anti-apoptosis processes. In addition, RKIP modulates other fundamental cell signaling pathways involving heterotrimeric G-proteins, keap1/nrf2, STAT3 and GSK [32]. Because of its interaction 
with multiple pathways, we consider RKIP as a master modulator of cellular processes. The amounts of RKIP proteins inside the cell can be regulated at multiple steps of expression, from pre-transcription of the gene to post-translation [33]. A plethora of metastatic solid tumors have RKIP downregulated or lost and experimental data suggests that that happens because of transcriptional or post-transcriptional regulation [24]. Here, we consider the that reduction on RKIP protein numbers happens because of repression of transcription.

Transcription of RKIP can be silenced by methylation of its promoter. Indeed, methylationspecific PCR (MSP) analysis has shown a sufficiently strong correlation between RKIP promoter methylation and low RKIP expression levels in cancer tumors, [24], like esophageal and gastric $[34,35]$. Histone modifications are also found as epigenetic mechanisms to regulate RKIP levels. Histone deacetylase inhibitors can increase RKIP transcripts [36,37]. Snail and BACH1 transcription factors can downregulate RKIP transcription by histone methyltransferases [38,39] and both are associated with epithelial-mesenchymal transition. Snail is a direct transcriptional repressor of the gene encoding the cell adhesion protein E-cadherin [40], and BACH1 is the basic leucine zipper protein expressed in mammalian tissue which positively regulates motility related genes that promote metastasis in breast cancers [41]. Expression of either Snail and BACH1 genes are self-repressed, and repressed by RKIP proteins. Additionally, BACH1 and RKIP combine into a bistable gene circuit that describe a switch for metastatic phenotype in tumor cellular population, as recently shown [39]. Taken together, those preliminary data indicate that RKIP expression levels may be used as a prognostic marker for survival probabilities and as a target for anti-metastatic treatment.

\subsection{An effective model for regulation of gene expression}

We interpret the gene as a source of gene products randomly switching between ON and OFF states. Synthesis of gene products takes place when the gene is ON at rate $k$ while at OFF state there is no synthesis. The rate of degradation of gene products is denoted by $\rho$. The gene switches from state ON to OFF, and from OFF to ON, with rates $h$, and $f$, respectively.

The aforementioned processes can be represented as a system of effective chemical reactions as given at Eqs. (1-4). We denote a gene product by $\mathcal{P}$ and its regulatory site by $\mathcal{R}$. In this manuscript, we consider the particular case of positive regulation of the gene by a transcription factor denoted by $\mathcal{T}_{\mathcal{F}}$.

$$
\begin{array}{rll}
\mathcal{R} & \stackrel{k}{\rightarrow} & \mathcal{R}+\mathcal{P}, \\
\mathcal{P} & \stackrel{\rho}{\rightarrow} & \oslash \\
\mathcal{R}+\mathcal{T}_{\mathcal{F}} & \stackrel{f}{\rightarrow} & \mathcal{R} \mathcal{T}_{\mathcal{F}}, \\
\mathcal{R} \mathcal{T}_{\mathcal{F}} & \stackrel{h}{\rightarrow} & \mathcal{R}+\mathcal{T}_{\mathcal{F}} .
\end{array}
$$

Eqs. (1-2) indicate, respectively, the gene product synthesis and degradation. The switching from OFF to ON state because of the binding of the activating protein, and the inverse transition caused by its unbinding, are respectively indicated in Eqs. (3-4). One may also consider the case of a transcription factor being a repressor. Then the effective reactions Eqs. (3-4) denote the ON to OFF and OFF to ON state transitions, with rates transformed as $f \rightarrow h$ and $h \rightarrow f$.

This system of effective reactions is very simple if we consider the complexity of regulation of gene expression in mammals. However, such a simplification enables the construction of exactly solvable quantitative models having a smaller number of parameters which we can use to investigate hypothetical treatment strategies before doing experiments. One example is the use of reduced dose multi-drug treatment targeting a functional network. A given drug has a specific half-life and the multiple drugs act on a system will combine as multi-timescale processes. Combining the dosage and application agenda of those multiple drugs to ensure both effectiveness and non-toxicity may be a hard task and 
a quantitative model may provide invaluable insights on the therapeutic design. Here we use the stochastic binary model for regulation of gene expression to investigate how the combination of two drugs modulates the dynamics of mRNA production.

\subsection{An effective model for investigating regulation of gene expression dynamics after treatment}

A biological interpretation of the effective model presented at Eqs. (1-4) is given. The rate $k$ is proportional to the inverse of the time interval between two consecutive bindings of RNAPolII to the promoter site. It implies on assuming that transcription starts after a negligible interval following RNAPolII binding. Alternatively, it might be interpreted as the inverse of the average interval between initiation of two subsequent transcription processes. The first case implies on assuming the availability of large amounts of RNAPolII and on interpreting $k$ as a consequence of the affinity between the promoter and RNAPolII. The second could be interpreted as the efficiency of the promoter on initiating transcription. Those two interpretations are not exclusive and, for simplicity, we refer to an increase on $k$ as an increase on the efficiency of the promoter.

The rates of switching $h$ and $f$ are the inverse of the average time of availability and unavailability of the promoter for the binding of the RNAPolII, respectively. The value of those rates will be determined by the binding of transcription factors to the regulatory regions of the gene. Despite the amounts of transcription factors change with time, it is fair to assume that they will remain constant during some sufficiently short intervals. Hence, during those short time intervals, we may employ the effective model of Eqs. (1-4). The values of the switching constants will reflect the balance resulting from the binding of activators, repressors, quenchers, pioneer factors and other regulatory elements interacting with the regulatory regions of the gene.

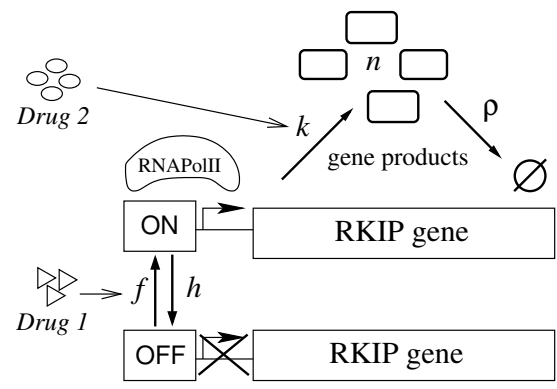

Figure 1. Drugs aiming kinetic rates of RKIP transcription. RNAPolII binds to the promoter region (when it is ON) of RKIP gene to synthesize mRNA (gene products). The switching between ON and OFF states is dependent of the regulatory components (proteins) surrounding the gene. Drug 1 targets an activator protein and aims to increase the time of exposition of the promoter for binding of RNAPolII. Drug 2 affects only the promoter by increasing its efficiency or the affinity of the promoter to the RNAPolII. A treatment consists of administering of a single or combinations drugs following a dose agenda.

Our model for regulation of gene expression suggests the design of multiple treatment strategies aiming to increase expression of RKIP gene. The model gives four kinetic rates which we may target by drugs. In the specific case of RKIP gene, one would attempt to increase $k$ and $f$ or to decrease $\rho$ and $h$. Those rates could be affected individually or collectively, in a coordinated manner, in case of a multi-drug therapy. In the scheme shown in Fig. 1 we consider the particular cases in which the treatment aims to: A) increase $f$; B) increase $k ; \mathbf{C}$ ) increase $f$ and $k$ concomitantly. We assume that a given treatment targets a rate exclusively, that is, the non-targeted rates will remain constant during treatment. Two mechanisms can be used for increasing RKIP expression levels:

i. Promoter demethylation Downregulation of RKIP has been associated with promoter methylation in many cancer types [34,35,42-44]. We suppose the use of a demethylation agent, namely, 5-Azacytidine (5-AzaC), to revert that (see scheme given in Fig. 2A in [18]). Consequently, expression levels of RKIP would be increased as previously tested in 
triple-negative breast cancer (TNBC) cell line SUM159 and esophageal cell lines TE- 1 and TE-13 [37,42].

ii. Transcription factors regulation The tumor environment is an inexhaustible source of signals which induce a change on the amounts of transcription factors regulating gene expression and, consequently, the phenotypes of tumor cells. For example, nitric oxide (NO) [45] affects tumor growth by downregulating functional quantities of NF- $\kappa$ B and SNAIL which, in reduced quantities, are associated with an increase on RKIP expression. Thus, one may propose a therapeutic strategy using NO donors, such as (Z)-1-[2-(2-aminoethyl)-N-(2ammonio-ethyl) amino] diazen-1-ium-1, 2-diolate (DETANONOate) [40,46,47], to keep a constant inhibition of the NF- $\kappa \mathrm{B} / \mathrm{SNAIL}$ loop. As a consequence, we expect an increase on the levels of transcripts of RKIP (see scheme in Fig. 2B in [18]).

Finding drugs specifically targeting promoter demethylation of RKIP gene or transcription factors regulating RKIP expression levels is a challenge beyond the scope of this manuscript. Therefore, as a strategy to introduce our methodology, to set a clinically relevant time-scale, and to show the difficulties of combining multiple drugs having multiple targets and time scales we consider non-specific drugs. In that case, we are only considering the effect of the drug on the gene that we describe. The cellular level effects will not be considered here as those would require a more complex approach in which the dynamics of expression levels of multiple potentially interacting genes would need to be considered.

\subsection{An approach for investigating treatment effects on RKIP expression dynamics}

Our treatment strategies aim to increase $f$ and $k$. We assume that the drug effectiveness on those quantities decays exponentially, and the kinetic rates will return to their pretreatment values. Hence, once treatment starts, the rates $f$ and $k$ become time-dependent with $f_{0}$ and $k_{0}$ being the OFF to ON and the synthesis rates, respectively, before treatment. We denote the effect of the dose on the value of a kinetic constant by $\xi$, where $0<\xi \leq 1$. We assume that at the maximal tolerated dose the effect of the drug is given by $\xi=1$ and that that dose raises the targeted rates to $f_{1}$ and $k_{1}$. That does not imply on the assumption of maximal efficiency of the drug effectiveness. Indeed, we consider that $\xi$ is the net effect of a given dose on its targeted rate. Hence, a given dose smaller than the maximal tolerated one will instantaneously affect its target rate towards $\xi_{a} f_{1}$ and $\xi_{b} k_{1}$, where $\xi_{a}$ and $\xi_{b}$ are non-linear functions of the dose. The time-dependent OFF to ON switching rate, $f(t)$, is

$$
f(t)=\left\{\begin{array}{cc}
f_{0}, & 0 \leq t<\tau_{1} \\
f_{0}+\left[f_{s}\left(\tau_{j}\right)-f_{0}\right] \mathrm{e}^{-\lambda_{a}\left(t-\tau_{j}\right)}, & \tau_{j} \leq t<\tau_{j+1}
\end{array}\right.
$$

and $k(t)$

$$
k(t)=\left\{\begin{array}{cc}
k_{0}, & 0 \leq t<\tau_{1} \\
k_{0}+\left[k_{s}\left(\tau_{j}\right)-k_{0}\right] \mathrm{e}^{-\lambda_{b}\left(t-\tau_{j}\right)}, & \tau_{j} \leq t<\tau_{j+1}
\end{array}\right.
$$

where $j=1, \ldots, J-1$ denotes the $j$-th drug application, $J$ is the amount of drug doses, $\tau_{j}$ is the time of the $j$-th drug application. At each application of the drug, the steady state condition is that of the untreated system, namely $f_{0}$ and $k_{0} .\left(\lambda_{a}, \lambda_{b}\right)$ denote the rates of exponential decay of the effect of the drugs on the rates $(f, k)$. As previously mentioned, we are considering that the effect of the drugs on their targeted rates is fast enough to be considered as instantaneous. Therefore, the values of the rates $f$ and $k$ immediately after the arrival of the $j$-th dose, respectively denoted by $f_{s}\left(\tau_{j}\right)$ and $k_{s}\left(\tau_{j}\right)$, will be considered as the initial conditions during the interval $\tau_{j} \leq t<\tau_{j+1}$. Note that the $j$-th dose adds up 
to the drug amounts that are remainders from previous applications. Hence, the initial condition at the time $\tau_{j}$ is written as:

$$
\begin{aligned}
& f_{s}\left(\tau_{j}\right)=f_{1} \sum_{i=1}^{j} \xi_{a}\left(\tau_{i}\right) \mathrm{e}^{-\lambda_{a}\left(\tau_{j}-\tau_{i}\right)}, \\
& k_{s}\left(\tau_{j}\right)=k_{1} \sum_{i=1}^{j} \xi_{b}\left(\tau_{i}\right) \mathrm{e}^{-\lambda_{b}\left(\tau_{j}-\tau_{i}\right)},
\end{aligned}
$$

where the dose may be calibrated to generate a differential effect at each time instant to prevent toxic accumulation of drug quantities.

\subsection{An approximate description of the stochastic binary gene expression dynamics with time-dependent kinetic rates}

The randomness of intracellular phenomena suggests a description of the treatment effects on expression of RKIP gene to be built in terms of an stochastic process. Fig 1 suggests the existence of two random variables that determine the state of the system: the gene state, being ON or OFF, and the number of gene products, denoted by $n$. The description of the dynamics of the state of the system is given in terms of a probability distribution

$$
\Pi\left(\alpha_{n}(t), \beta_{n}(t)\right),
$$

where $\alpha_{n}(t)$, or $\beta_{n}(t)$, denote the probability of finding $n$ proteins at time $t$ when the gene is $\mathrm{ON}$, or OFF, respectively. A master equation governing the probability distribution for an externally regulated gene can be written as:

$$
\begin{aligned}
\frac{d \alpha_{n}}{d t} & =k(t)\left(\alpha_{n-1}-\alpha_{n}\right)+\rho\left[(n+1) \alpha_{n+1}-n \alpha_{n}\right]-h \alpha_{n}+f(t) \beta_{n}, \\
\frac{d \beta_{n}}{d t} & =\rho\left[(n+1) \beta_{n+1}-n \beta_{n}\right]+h \alpha_{n}-f(t) \beta_{n},
\end{aligned}
$$

where $h$ and $\rho$ are constants and Eqs. (5) and (6) give $f(t)$ and $k(t)$. The existence of time-dependent coefficients turns hard for one to solve Eqs. (10) and (11) analytically, and some numerical method needs to be employed. The interpretation of the master equation is built in terms of the coefficients $k(t), \rho, f(t)$, and $h$ as presented on the description of the Eqs. (1-4) and the cartoon shown on Fig. 1.

Here we propose to one to approximate the dynamics of the kinetic rates $f(t)$ and $k(t)$ of Eqs. (5) and (6) as piecewise functions assuming constant values during sufficiently short time intervals ensuring that the difference between the exact and approximated values lies within a given error size. Then, we may consider the model for constant kinetic rates during an interval which is exactly solvable. In that case, the initial condition of an interval is the final condition on its previous neighbor.

\subsection{An exactly solvable model for benchmarking cancer treatment aiming to modulate gene expression levels}

The master equation (10) and (11) with constant coefficients has been already proposed, and it is fully solvable at the stationary [16] and time-dependent regimes [17]. The existence of exact solutions enables one to calculate the time-dependent functions governing the first and the second moment of the number of gene products [18]. Here we write the explicit expressions governing the dynamics of the average number of gene products, $\langle n\rangle(t)$ and the standard deviation, $\sigma(t)=\sqrt{\left\langle n^{2}\right\rangle(t)-\langle n\rangle^{2}(t)}$. We use Eqs. (1-4) and define the following constants:

$$
N=\frac{k}{\rho} ; \quad A_{s}=\frac{f}{f+h} ; \quad \epsilon=\frac{f+h}{\rho},
$$

which are, respectively, the steady state expected number of gene products in case of a gene being fully $\mathrm{ON}$, (we call it maximal mRNA number $N$ ); the steady state probability for 
the gene to be $\mathrm{ON}\left(A_{S}\right)$; the ratio of the gene switching rate between $\mathrm{ON}$ and $\mathrm{OFF}$ states to the degradation rate of the gene products (we call it switching speed $\epsilon$ ).

The average number of mRNAs and the standard deviation at the steady state regime are, respectively, denoted by $\langle n\rangle_{s}$ and $\sigma_{s}$. We write them as functions of the parameters of Eq. (12):

$$
\begin{aligned}
\langle n\rangle_{s} & =A_{s} N \\
\sigma_{s}^{2} & =\langle n\rangle_{s}\left(1+N \frac{1-A_{s}}{1+\epsilon}\right)
\end{aligned}
$$

The dynamics of the average and standard deviation is denoted by $\langle n\rangle(t)$ and $\sigma(t)$, respectively, and we write:

$$
\begin{aligned}
\langle n\rangle(t) & =\langle n\rangle_{s}+Y \mathrm{e}^{-\epsilon \rho t}+V \mathrm{e}^{-\rho t}, \\
\sigma^{2}(t) & =\sigma_{s}^{2}+U_{1} \mathrm{e}^{-\epsilon \rho t}+V \mathrm{e}^{-\rho t}+W_{1} \mathrm{e}^{-(1+\epsilon) \rho t}+X_{1} \mathrm{e}^{-2 \rho t}-Y^{2} \mathrm{e}^{-2 \epsilon \rho t} .
\end{aligned}
$$

The coefficients of the exponentials are integration constants given on the Appendix A.2. These solutions are obtained from a system of ordinary differential equation coupling the moments $A,\left\langle n_{\alpha}\right\rangle$, and $\left\langle n^{2}\right\rangle$ which exact forms are given on Appendix A.3.

Eqs. (15) and (16) enable us to compute the evolution of the average number of products from RKIP gene and its standard deviation using a piecewise representation of the time-dependent rates $f(t)$ and $k(t)$ as we show on next section.

The decaying rate to steady state of both $\langle n\rangle(t)$ (Eq. 15) and $\sigma(t)$ (Eq. 16) can be established in terms of $\epsilon$. For $\epsilon \gg 1$, the terms $e^{-\epsilon \rho t}, e^{-(\epsilon+1) \rho t}$ become null much faster than the term $e^{-\rho t}$, which hence determines the approaching to steady state. Alternatively, for $\epsilon \ll 1$, the term $e^{-\rho t}$ will govern lifetime of the dynamical regime, that is, the regime during which $\langle n\rangle(t)$ and $\sigma(t)$ are varying with time.

Additionally, one may notice that $\epsilon$ also reflects the ratio of the gene switching frequence to the degradation rate, the characteristic times of the two processes being coupled. Eq. (14) indicates that $\sigma_{S}^{2} \rightarrow\langle n\rangle_{S}$ when $\epsilon \gg N>1$. That coincides with the decaying to steady state being determined by the gene product degradation rate, as it happens on a Poisson process. On the other hand, when the gene switching decaying rate to steady state is prevalent, for $\epsilon \ll 1, \sigma_{s}^{2}$ will have larger values. Then, the gene switching will have a stronger effect on the fluctuations of the number of gene products.

\subsection{Parameter values and conditions for treatment simulations}

We describe simulation conditions for the three treatment scenarios. We denote the degradation rate of DETANONOate (acting on $f$ ) and 5-AzaC (acting on $k$ ) by $\lambda_{a}$ and $\lambda_{b}$, which values are $\lambda_{a}=0.05 \mathrm{~h}^{-1}$ [48] and $\lambda_{b}=0.25 \mathrm{~h}^{-1}$ (DB00928 entry at DrugBank [49]). The response generated on the kinetic rates by treatment at maximal tolerated dose is indicated by $\xi_{a}=\xi_{b}=1$. For a single dose, we assume that the drugs change the values of their targeted rates instantaneously after application, and for maximum tolerated dose, $f \rightarrow f_{1}$ and $k \rightarrow k_{1}$, as indicated by Eqs. (7) and (8), respectively. Note that the maximum tolerated dose is drug specific and its effect on each kinetic rate can be denoted by the value of $\xi$.

The gene product of RKIP is assumed to be the mRNAs, which degradation rate is $\rho=0.17 \mathrm{~h}^{-1}$ [50]. The pre-treatment condition, occurring for $0 \leq t<\tau_{1}$, is not be shown because we assume it as a stationary state so that we set $\tau_{1}=0$. The pre-treatment condition is characterized by a low average copy number of RKIP mRNA's (here chosen as $\langle n\rangle_{0}=10$ ). We arbitrarily assume that in pre-treatment condition, the levels of mRNA's are $8 \times$ below what would be expected to be found in a non-metastatic cell. Hence, the treatment is assumed to be successful if it drives the probability of finding less than $\langle n\rangle_{T} \approx 80$ mRNAs to a negligible value. That is an important requirement to minimize heterogeneity on treatment response. 
The randomness of intracellular processes causes a variability on treatment response even under the hypothetical condition in which all individuals of a population of genetically identical cells absorb the same drug dosage. The heterogeneity of the response can be quantified by the standard deviation of the number of gene products. Hence, we first set $\langle n\rangle_{1}=100$ as the expression level aimed by treatment. This value is chosen assuming that a gene expressing an average of $100 \mathrm{mRNAs}$ in a Poisson regime has the threshold value $80=\langle n\rangle_{1}-2 \sigma_{1}$. Here, $\sigma_{1}=\sqrt{\langle n\rangle_{1}}$ is the standard deviation of a Poisson distribution having $\langle n\rangle_{1}$ as its average.

The dynamics of $f$ and $k$ are described by Eqs. (5) and (6). We approximate them as piecewise functions and compute the error using integrals of the exponential decays along each subinterval. Then, we set the length of each subinterval by fixing its error. The piecewise approximation enables the use of $\langle n\rangle(t)$ (Eq. 15) and $\sigma(t)$ (Eq. 16) to describe the expression of RKIP.

A single dose will not be sufficient for keeping $\langle n\rangle_{1} \approx 100$ because of the exponential decay of the drug effect on the kinetic constants (as it will be shown on graphs A-E of Figs. 3-5). Hence, multiple doses are necessary and we determine the intervals between applications of DETANONOate and 5-AzaC as, respectively, $10 \mathrm{~h}$ and $4 \mathrm{~h}$. These numbers were chosen to ensure that $\langle n\rangle(t) \approx 100$ during a sufficiently long time interval and their choice is based on the degradation rates of each drug.

The treatment changes the dynamical properties of the gene switching. When we consider DETANONOate, the pre-treatment probability of finding the gene $\mathrm{ON}$ is $A_{0}=\frac{f_{0}}{f_{0}+h}$. The drug dose delivered at $\tau_{j}$ causes $f_{0} \rightarrow f_{s}\left(\tau_{j}\right)$ instantaneously as defined at Eq. (7). Then, the aimed steady state probability for the gene to be ON is $A_{1}=\frac{f_{s}\left(\tau_{j}\right)}{f_{s}\left(\tau_{j}\right)+h}$. The preand instantaneously post - treatment value of the gene switching frequence is, respectively, $\epsilon_{0}=\frac{f_{0}+h}{\rho}$ and $\epsilon_{1}=\frac{f_{s}\left(\tau_{j}\right)+h}{\rho}$. When we consider 5 - $A z a C$ the probability of finding the gene $\mathrm{ON}$, given by $A_{0}=\frac{f_{0}}{f_{0}+h}$, and gene switching frequence $\epsilon_{0}=\frac{f_{0}+h}{\rho}$, will both remain constant while the value of $k_{0} \rightarrow k_{s}\left(\tau_{j}\right)$ instantaneously after drug application at $\tau_{j}$ - see Eq. (8) - such that $N_{0}=\frac{k}{\rho} \rightarrow N_{1}=\frac{k_{s}\left(\tau_{j}\right)}{\rho}$.

\section{Results}

We simulate three treatment scenarios: 1 . denotes the effect of maximum tolerated dose of DETANONOate; 2 . denotes the effect of maximum tolerated dose of 5-AzaC; 3 . denotes the effect of fractionary doses of the two drugs together.

The treatment response is quantified in terms of the time for the average value to reach the threshold and the standard deviation. These two quantities are strongly dependent of the values of $\epsilon_{0}$, as we will show on next subsections. The values of $\rho, \lambda_{a}$, and $\lambda_{b}$ are assumed to remain constant during treatment. All rates $(k, f, h)$ are given in $\mathrm{h}^{-1}$.

The values of the parameters are selected for simulating treatment conditions which probability distributions governing expression of pre-treated cells indicate qualitatively distinguishable steady state regimens as recently classified [19]. Fig. 2 shows pre-treatment (and aimed post-treatment) steady state probability distributions governing the numbers of RKIP mRNAs in red (and green). The values of the parameters in each row are set to ensure that graphs $\mathbf{A}$ indicate bimodal distributions, graphs $\mathbf{B}$ are distributions close to the limit of the bimodal regime, graphs $\mathbf{C}$ indicate the regime in which the probabilities are table-shaped for $A_{0}=A_{1}=0.5$ (as indicated in graph $\mathbf{C 2}$ ), graphs $\mathbf{D}$ indicate the quasi-Poisson distributions, and graphs $\mathbf{E}$ denote the bursting limit. The curves of the steady state probability distributions of finding $n$ mRNAs, within the Fig. 2, are computed by Eq. (A1) and it is denoted by $\tilde{\phi}_{n}$ in the Appendix A.1.

The parameters of the pre- and post-treatment probability distributions are fixed such that the steady state average number of mRNA's will be $\sim 10$ and $\sim 100$, respectively. The bimodal distributions indicate that the mRNAs synthesized while the gene is ON will degrade fastly after the switching to the OFF state. For the table-shaped and quasi- 

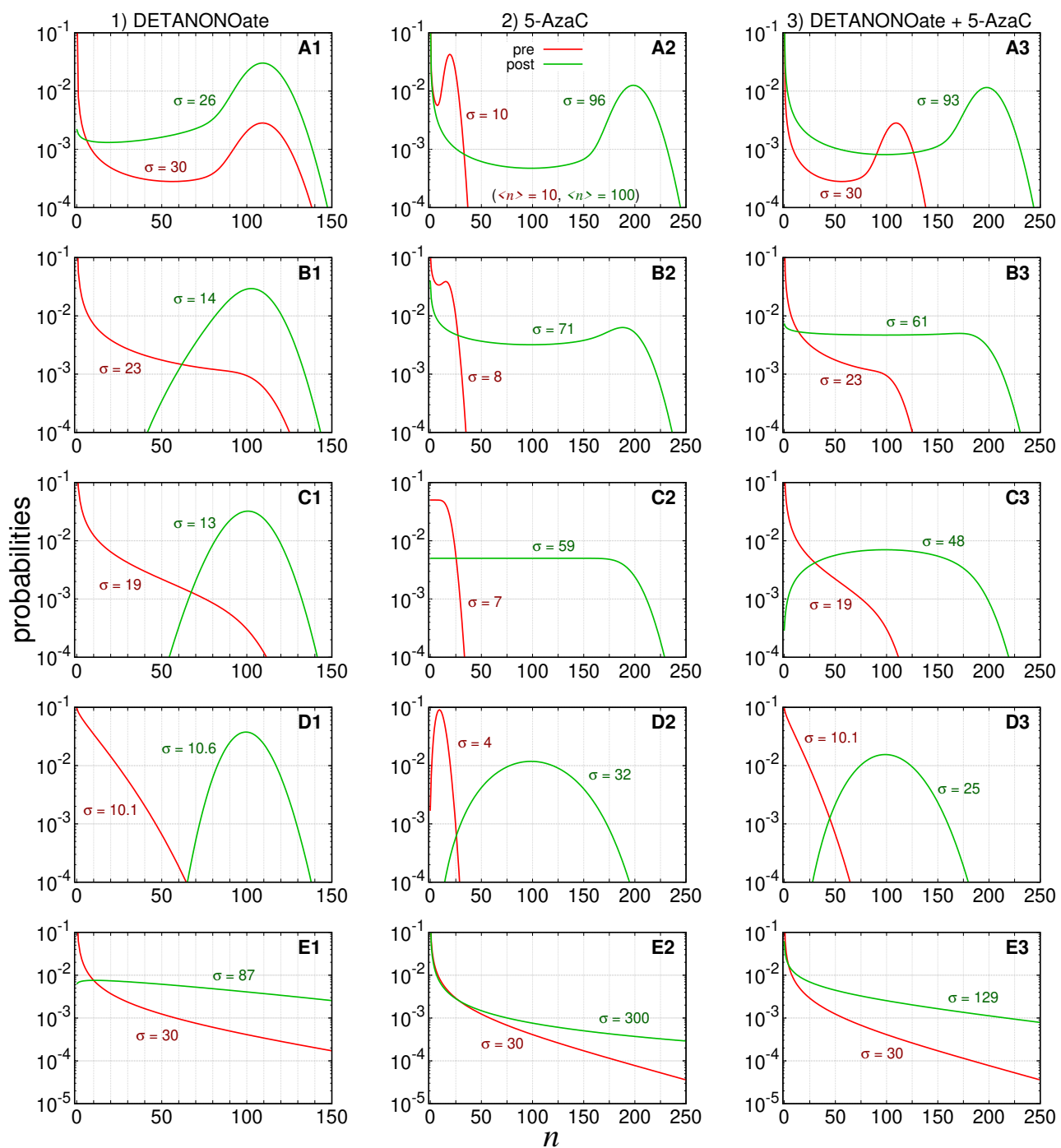

Figure 2. Probability distributions. The red lines indicate pre-treatment probabilities while the green lines indicate the aimed probabilities governing the post-treatment number of gene products. The post-treatment distribution parameters were set aiming to increase the average number of transcripts to be $\approx 100$. We disregarded the effect of the new parameter values on the variances, i.e., the heterogeneity of treatment response. We consider five initial conditions for the gene switching speed, $\epsilon_{0}=(0.1,1,2,10,10)$, arranged in rows and indicated by $\mathbf{A}-\mathbf{E}$ in the labels of respective graphs. Graphs $\mathbf{D}$ and $\mathbf{E}$ have the same value for $\epsilon_{0}$ but represent different because of the differing values of $A_{0}$. The distributions for the treatment designs are arranged in columns: 1. DETANONOate aiming the $f$ rate, 2. 5-AzaC aiming the $k$ rate, and 3. both drugs aiming both $f$, and $k$ rates, simultaneously.

Poissonian limits, the switching between ON-OFF states are, respectively, slow and fast enough to ensure that the mRNA degradation is compensated by its synthesis to generate the specific distributions. The burst limit is characterized by very short ON states during which the synthesis is very efficient while the OFF states duration is proportionally very long.

On next subsections we present the results of simulations of the treatment response dynamics considering the five pre-treatment conditions shown in Fig. 2. The Figs (3)-(5) show response after application of a single (or multiple) doses on graphs labeled as A-E (or F-J) followed by the number indicating the treatment scenario $(1,2$ or 3$)$. The pre-treatment conditions have $\epsilon_{0}=(0.1,1,2,10,10)$. The black dashed lines indicate the aimed average value after treatment. The green lines indicate $\langle n\rangle(t)$ and the red lines indicate the values of $\langle n\rangle(t) \pm \sigma(t)$. The parameters of the treatment are set to enable the average number of 
gene products to reach the post-treatment regime shown in Fig. 2 for each set of parameter values.

\subsection{Treatment aiming the OFF to ON gene state switching rate}

Fig. 3 shows a simulation of the dynamics of the average number of mRNAs and its standard deviation resulting from a change on $f$ after introduction DETANONOate. The absolute error of each subinterval of the piecewise approximation for $f(t)$ is $1 \times 10^{-4}$. We assume the rates $(k, h, \rho)$ being constant during treatment. For obtaining graphs A1-D1 and F1-I1 we set $\left(k_{0}, N_{0}\right)=\left(k_{1}, N_{1}\right)=(18.5,110)$ and $\left(A_{0}, A_{1}\right)=(0.09,0.9)$. Graphs E1 and J1 have $\left(k_{0}, N_{0}\right)=\left(k_{1}, N_{1}\right)=(166.7,1000)$ and $\left(A_{0}, A_{1}\right)=(0.01,0.1)$ to ensure the bursting gene expression regime during the pre-treatment stage. On graphs A1-E1 (or F1-J1) we set $f_{0}=(0.0015,0.015,0.03,0.15,0.017)$ and $f_{1}=(0.14,1.37,2.73,13.65,0.18)$, such that, $h=$ $(0.015,0.15,0.3,1.52,1.65)$

and $\epsilon_{1}=(0.9,9.1,18.2,91,11)$ when we the given values of $A_{0}, A_{1}$ and $\rho$. The maximum tolerated dose is considered to cause the steady state $\mathrm{ON}$ state probability to be multiplied by ten, as indicated by the values of $A_{0}$ and $A_{1}$.
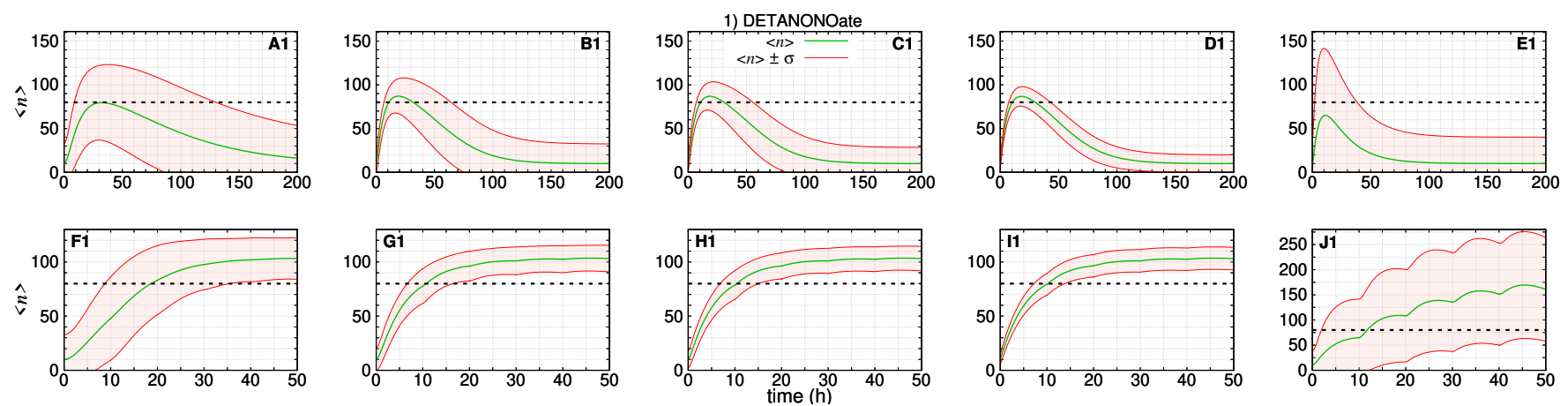

Figure 3. The dynamics of the number of RKIP mRNA (green solid line) and their standard deviation (red solid line) along the time for treatment aiming $f$ kinetic rate. The black dashed line at 80 indicates an arbitrary threshold on the number of RKIP mRNA. Graphs A1-E1 show single doses of drug with different progressive values to $\epsilon_{0}$ (shown within respective graph). Graphs F1-J1 show the same initial conditions that Graphs A1-E1 for 5 doses with time interval of $10 \mathrm{~h}$.

The first and second rows of graphs of Fig. 3 show the simulation of the dynamics of expression after one and five drug doses, respectively, under 5 pre-treatment conditions.

Graphs A1 and B1 show the dynamics of response of the slow switching gene, a regime of synthesis of mRNAs which numbers are governed by a bimodal distribution at the steady state. For $\epsilon_{0}=0.1$ we have the slowest treatment response and return to pre-treatment conditions. The average number of mRNAs reaches a maximum of $\sim 80$ after $\sim 30 \mathrm{~h}$. The standard deviation is the second largest. Graphs A1 to D1 show the average mRNA number reaching or crossing the threshold in our simulations, the time for the average number to reach a maximum when $\epsilon_{0} \geq 1$ is $\sim 20 \mathrm{~h}$ and the noise of the response decreases as we increase $\epsilon_{0}$. The exception is shown on graph E1 where the noise is the largest and the time for the average number to reach a maximum is $\sim 10 \mathrm{~h}$. Note that in this case the average number does not cross the threshold and the lower line $\langle n\rangle(t)-\sigma(t)$ does not appear.

The response to multi-doses treatment is shown in graphs F1 to J1. The interval between doses was chosen to enable a sigmoidal-like response characterizing two distinct levels of expression with the average number increasing from 10 to at least $\sim 100$. Graph F1 shows that the slow switching gene also causes the slowest response as $\langle n\rangle(t)$ crosses the threshold after $\sim 18 \mathrm{~h}$. The curve for $\langle n\rangle(t)-\sigma(t)$ also crosses the threshold after $\sim 35 \mathrm{~h}$, which ensures a less heterogeneous response to treatment. Graphs G1 to I1 show the simulations for increasing values of the gene switching. Those simulations show that $\langle n\rangle(t)$ crosses the threshold after $\sim 10 \mathrm{~h}$, and the curves $\langle n\rangle(t)-\sigma(t)$ reach higher values, which establishes the response to treatment having the minimal heterogeneity. Graph J1 
shows the response of a bursty gene which $\langle n\rangle(t)$ crosses the threshold after $\sim 12 \mathrm{~h}$ and reaches a maximal value of $\sim 170$. However, this regime causes the noisiest response as indicated by $\langle n\rangle(t)-\sigma(t)$ not crossing the threshold. Note also that there are some "bumps" because the effect of the single dose is close to the maximum by the time of the next drug application. It is possible to prevent the bumps but we decided to show them to demonstrate that the time interval between drug dosages also requires one to consider the dynamics of the targeted gene.

\subsection{Treatment aiming the RKIP $m R N A$ synthesis rate}

Fig. 4 shows the dynamics of the average number of RKIP's mRNAs and its standard deviation under treatment with 5-AzaC. The absolute error of each subinterval of the piecewise approximation for $k(t)$ is $1 \times 10^{-4}$. We assume that the rates $(f, h, \rho)$ remain constant during treatment, which implies on $A_{0}=A_{1}$ and $\epsilon_{0}=\epsilon_{1}$ also being constant. For graphs A2-D2 and F2-I2 we set $\left(k_{0}, N_{0}, k_{1}, N_{1}\right)=(3.3,20,33,200), A_{0}=0.5$ and $f=h=(0.008,0.08,0.17,0.83)$. For graphs $\mathbf{E 2}$ and $\mathbf{J} 2$ we set $\left(k_{0}, N_{0}, k_{1}, N_{1}\right)=$ $(166.7,1000,1667,10000), A_{0}=0.01$ and $(f, h)=(0.017,1.65)$.
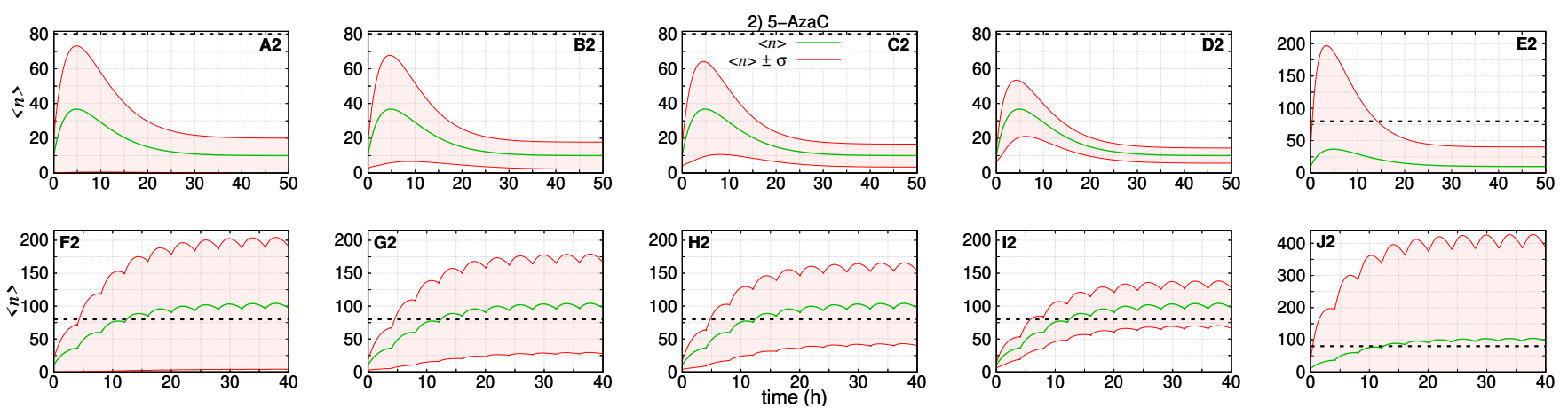

Figure 4. The dynamics of the number of RKIP mRNA (green solid line) and their standard deviation (red solid line) along the time for treatment aiming $k$ kinetic rate. The black dashed line at 80 indicates an arbitrary threshold on the number of RKIP mRNA. Graphs A2-E2 show single doses of drug with different progressive values to $\epsilon_{0}$ (shown within respective graph). Graphs F2-J2 show the same initial conditions that Graphs A2-E2 for 10 doses with time interval of 4 h.

The first and second rows of graphs of Fig. 4 show the simulation of the dynamics of expression after one and ten drug doses, respectively, under 5 pre-treatment conditions.

The treatment do not affect either $\epsilon$ or $\rho$, the decaying rates of the system are not affected. Then, graphs A2 to E2 show that the average number of mRNAs will reach the maximum ( $\sim 35$ molecules) after similar intervals of $\sim 5 \mathrm{~h}$ and reach pre-treatment conditions after $\sim 40 \mathrm{~h}$. Graph A2 shows the condition with the second largest standard deviation. Graphs A2 to D2 show that the noise of response decreases with the increase of $\epsilon_{0}$. The bursty regime shown in graph E2 leads to the noisiest response. Indeed, $\langle n\rangle(t)-\sigma(t)$ remains negative while $\langle n\rangle(t)+\sigma(t)$ exceeds the threshold more than $2 \times$.

The response to multi-doses treatment is shown in graphs F2 to J2. The interval between doses was chosen to enable a sigmoidal-like response with the average number reaching at least 100. All graphs show that $\langle n\rangle(t)$ cross the threshold after $\sim 10 \mathrm{~h}$. That is because the response to treatments and interval between drug application is the same in all scenarios. Besides, since both $\epsilon_{0}$ and $\rho$ are not affected, the decaying rates to pretreatment conditions are not affected. In all scenarios, the curves for $\langle n\rangle(t)-\sigma(t)$ do not cross the threshold because the treatment do not increase $\epsilon_{0}$ that would cause a reduction on the noise in mRNA synthesis in a given scenario. However, graphs F2 to I2 show that $\langle n\rangle(t)-\sigma(t)$ reaches higher maximum values as $\epsilon_{0}$ increases. Graph $\mathbf{J} 2$ shows the response in case of transcriptional bursts, which leads to the most heterogeneous response to treatment as indicated by the curve $\langle n\rangle(t)+\sigma(t)$ reaching the highest maximum. 


\subsection{Treatment with the two drugs concomitantly}

Fig. 5 shows the dynamics of the average number of mRNAs and its standard deviation under treatment with both drugs: DETANONOate and 5-AzaC. The absolute errors of each subinterval of the piecewise approximation for $f(t)$ and $k(t)$ are $1 \times 10^{-4}$ for both. respectively. The rates $(h, \rho)$ remain constant during treatment. For graphs A3-D3 (or F3-I3), we set $\left(k_{0}, N_{0}, k_{1}, N_{1}\right)=(18.5,110,33,200),\left(A_{0}, A_{1}\right)=(0.09,0.5)$, $f_{0}=(0.0015,0.015,0.03,0.15)$ and $f_{1}=h=(0.015,0.15,0.3,1.5)$. For graphs E3 and J3 we set $\left(k_{0}, N_{0}, k_{1}, N_{1}\right)=(167,1000,333,2000),\left(A_{0}, A_{1}\right)=(0.01,0.05), f_{0}=0.017$, $f_{1}=0.087$ and $h=1.65$. The values of $A_{0}, A_{1}$ and $\rho$ results in $\epsilon_{1}=(0.18,1.8,3.6,18,10.4)$ on graphs A3-E3 (or F3-J3).
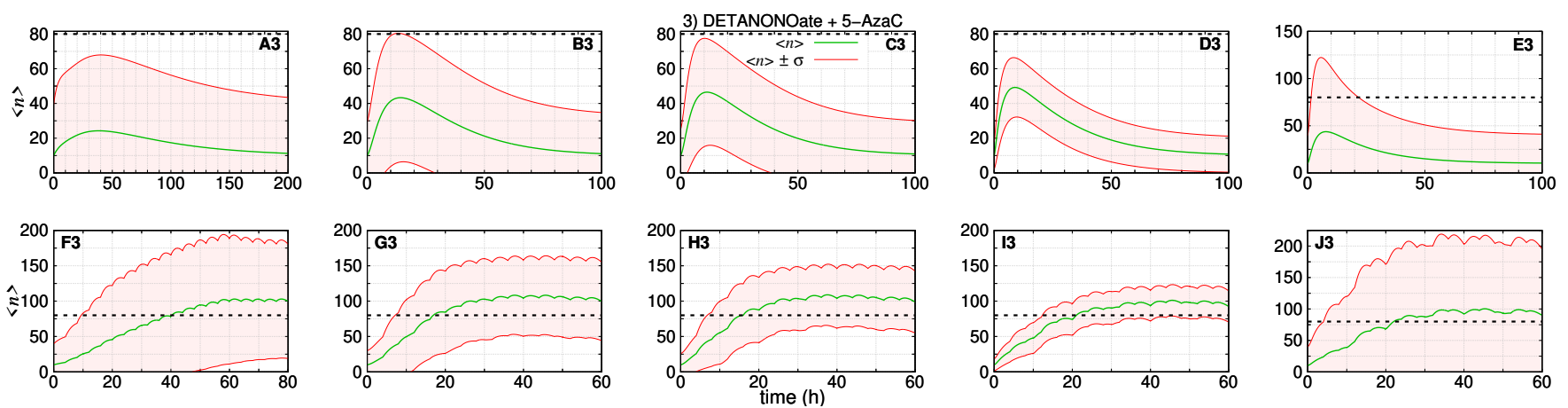

Figure 5. The dynamics of the number of RKIP mRNA along the time for treatment with two drugs that aiming $f$ and $k$ kinetic rates are shown in green solid line and their standard deviation in red solid line. The threshold for RKIP is 80 and it is shown within graphs as black dashed line. Graphs A3-E3 show the single doses of both drugs for different progressive values to $\epsilon_{0}$ (indicate within each graph). Graphs F3-J3 show the same initial conditions that Graphs A3-E3 for multiple fractional doses of drug that target $f$ (or $k$ ) with time interval of $10 \mathrm{~h}$ (or $4 \mathrm{~h}$ ), in order, with 8 (or 20) doses in Graph F3 and 6 (or 15) doses in Graph G3-J3. The agenda of doses is shown in Table 1, where the number of doses of each drug and their respective fractions, $\xi_{a}$ and $\xi_{b}$ are detailed.

Fig. 5 shows the simulations of the dynamics of response to treatment. Graphs of first row indicate the results of simultaneous application of a single dose of both drugs. Second row shows the result of application of multiple doses with drug targeting $f$ (or $k$ ) being applied every $10 \mathrm{~h}$ (or $4 \mathrm{~h}$ ) accordingly with agenda in Table 1.

Graph A3 shows the dynamics of treatment response when the initial condition is set for a slow switching gene. The average number reaches a maximum of $\sim 25$ at $t \sim 35 \mathrm{~h}$. The maximum of $\langle n\rangle(t)+\sigma(t)$ is $\sim 70$, a highly heterogeneous response if we consider that the maximum standard deviation is equal to the maximum average, that is, it is a superPoissonian regime of gene transcription. The return to pre-treatment is slow as indicated by the decay of the average after $\sim 200 \mathrm{~h}$. In graphs B3, C3, and D3, the maximum average number of mRNAs is $\sim 40, \sim 45$, and 50, respectively, reached after $15 \mathrm{~h}, 12 \mathrm{~h}, 8 \mathrm{~h})$. The curves $\langle n\rangle(t)-\sigma(t)$ and $\langle n\rangle(t)+\sigma(t)$ become closer with the increase of $\epsilon_{0}$ as shown from A3 to D3. The burst limit is different and, despite the high value of $\epsilon_{0}$, has larger noise as shown in graph E3. At this limit, $\langle n\rangle(t)$ maximum is $\sim 45$ reached after $\sim 8$ h. $\langle n\rangle(t)+\sigma(t)$ reaches a maximum of $\sim 120$, indicating the noisiest response. For all pre-treatment conditions, the average number has not crossed the threshold.

The response to multi-doses treatment is shown in graphs F3 to J3. Table 1 shows the sequences of applications of a given drug in fractions of maximal tolerated dose, $\xi_{a}$ and $\xi_{b}$. The values were chosen aiming that the total amount of drugs is reduced in comparison with the single drug treatment. We also attempt to ensure a sigmoidal-like response such that the average number will reach at least $\sim 100$. Graph F3 shows the dynamics of response for 8 (or 20) doses of drug targeting $f$ (or $k$ ) with $\xi_{a}$ and $\xi_{b}$ ranging from 0.9 to 0.5 (details in Table 1). The agenda enables a reduction of $20 \%$ in comparison with application of full doses $\left(\xi_{a}=\xi_{b}=1\right)$. Because the pre-treatment gene is in a slow switching regime that leads to the slowest response as $\langle n\rangle(t)$ crosses the threshold after $\sim 40 \mathrm{~h}$. It also has 
Table 1. The fractions $\xi_{a}$ and $\xi_{b}$ of maximal tolerated dose for treatment agendas of pre-treatment conditions in Fig. 5

\begin{tabular}{cccccc}
\hline \multirow{2}{*}{ Graph } & \multicolumn{2}{c}{ Sequence of number of doses $\times$ fraction for } & & \multicolumn{2}{c}{ Cumulative reduction in } \\
\cline { 2 - 3 } \cline { 5 - 6 } & $\xi_{a}$ & $\xi_{b}$ & & $\xi_{a}$ & $\xi_{b}$ \\
\hline F3 & $3 \times 0.9 ; 4 \times 0.8 ; 1 \times 0.5$ & $5 \times 0.9 ; 10 \times 0.8 ; 5 \times 0.7$ & & $20 \%$ & $20 \%$ \\
G3 & $3 \times 0.8 ; 1 \times 0.7 ; 2 \times 0.5$ & $5 \times 0.8 ; 10 \times 0.75$ & & $32 \%$ & $23 \%$ \\
H3 & $3 \times 0.8 ; 1 \times 0.7 ; 2 \times 0.5$ & $5 \times 0.8 ; 10 \times 0.75$ & & $32 \%$ & $23 \%$ \\
I3 & $4 \times 0.7 ; 1 \times 0.6 ; 1 \times 0.5$ & $15 \times 0.7$ & & $35 \%$ & $30 \%$ \\
J3 & $3 \times 0.7 ; 1 \times 0.65 ; 2 \times 0.6$ & $5 \times 0.7 ; 5 \times 0.65 ; 5 \times 0.6$ & & $29 \%$ & $35 \%$ \\
\hline
\end{tabular}

a high noise as it can be noticed by the values of $\langle n\rangle(t) \pm \sigma(t)$. Graphs $\mathbf{G}$ to J3 show the simulations of the response for 6 (or 15) doses of drugs $a$ (or $b$ ) with $\xi_{a}$ and $\xi_{b}$ ranging from 0.8 to 0.5 (see the Table 1). The cumulative reduction in full doses range from $29 \%$ to $35 \%$ (or from $23 \%$ to $35 \%$ ) in $\xi_{a}$ (or $\xi_{b}$ ). Graphs G3 to I3 show that $\langle n\rangle(t)$ crosses the threshold after $\sim 15 \mathrm{~h}$. The curves $\langle n\rangle(t) \pm \sigma(t)$ become closer as the value of $\epsilon$ increases which indicates the direction to design treatment strategies with reduced response heterogeneity. Graph J3 shows the bursty pre-treatment condition. Here, $\langle n\rangle(t)$ crosses the threshold after $\sim 22 \mathrm{~h}$ and $\langle n\rangle(t)+\sigma(t)$ reaches $\sim 200$, which indicates the noisiest response.

\subsection{Enhancing ineffective treatments aiming all kinetic rates of model}

Response to enhanced treatment is shown in Fig. 6 for five qualitatively different initial conditions aiming at optimal distribution. The hypothetical drug cocktail targets all rates of the model and the we reach an optimal response time and heterogeneity reduction for all qualitatively distinguishable initial conditions which in previous subsections leaded to unsatisfactory results. The pre-treatment distributions are shown by the red curves within the graphs in Fig. 2: A2, B3, C2, D3, E3 which, respectively, describe a bimodal, bimodal limit, table shaped, quasi-poissonian and burst steady state regimes of gene transcription. Our goal was to keep the average numbers of mRNAs around 100 and their fluctuations above the threshold. The decaying rates of the drugs affecting the drugs $k, \rho$, $f$, and $h$ are denoted by $\lambda_{i}$, where $i$ indicates the rate targeted by the drug. The decaying rate of the the drugs is fixed in $\mathrm{h}^{-1}:\left(\lambda_{k}, \lambda_{\rho}, \lambda_{f}, \lambda_{h}\right)=(0.25,6,0.05,0.053)$, and maximal tolerated dose of drug $i$ is denoted by $\left(\xi_{i}=1\right)$. The values of $\lambda_{f}$ and $\lambda_{k}$ are the same used previously from DETANONOate and 5-AzaC, respectively, while $\lambda_{\rho}$ and $\lambda_{h}$ values were set based on mRNA-microRNA binding duration time [51] and $h$ in BACH1 half-life [52]. The time-dependence of $h(t)$ and $\rho(t)$ is described following the same framework used for $k(t)$ and $h(t)$ - see Eqs. (5-8). To obtain the dynamics of the average number of mRNAs and of the standard deviation, we extend the previous formulation of the master equation of Eqs. (10) and (11) to all kinetic rates.

The standard deviation of the post-treatment probability distribution is set to be

$$
\sigma_{1}=\sqrt{N_{1} A_{1}\left[1+N_{1} \frac{\left(1-A_{1}\right)}{1+\epsilon_{1}}\right]},
$$

which has a local minimum at $N_{1} \rightarrow 0$ and a local maximum at

$$
A_{1}=\frac{1}{2}\left(1+\frac{1+\epsilon_{1}}{N_{1}}\right)
$$

where $N$ is the maximal mRNA number, $A$ is the steady state probability for the gene to be $\mathrm{ON}, \epsilon$ is the gene switching speed (Eq. 12) and the subscript 1 indicates aimed post-treatment parameter values. A strategy to reduce $\sigma_{1}$ is to keep $N_{1} \gtrsim 1+\epsilon_{1}$ (or $\left.k_{1} \gtrsim f_{1}+h_{1}+\rho_{1}\right)$, which ensures that $\sigma_{1}$ approaches the local maximum as $A_{1} \rightarrow 1$. The reduction of $\sigma_{1}$ values depends on the mean number $\langle n\rangle_{1}$ desired, so that $\langle n\rangle_{1} \approx A_{1}\left(1+\epsilon_{1}\right)$. 

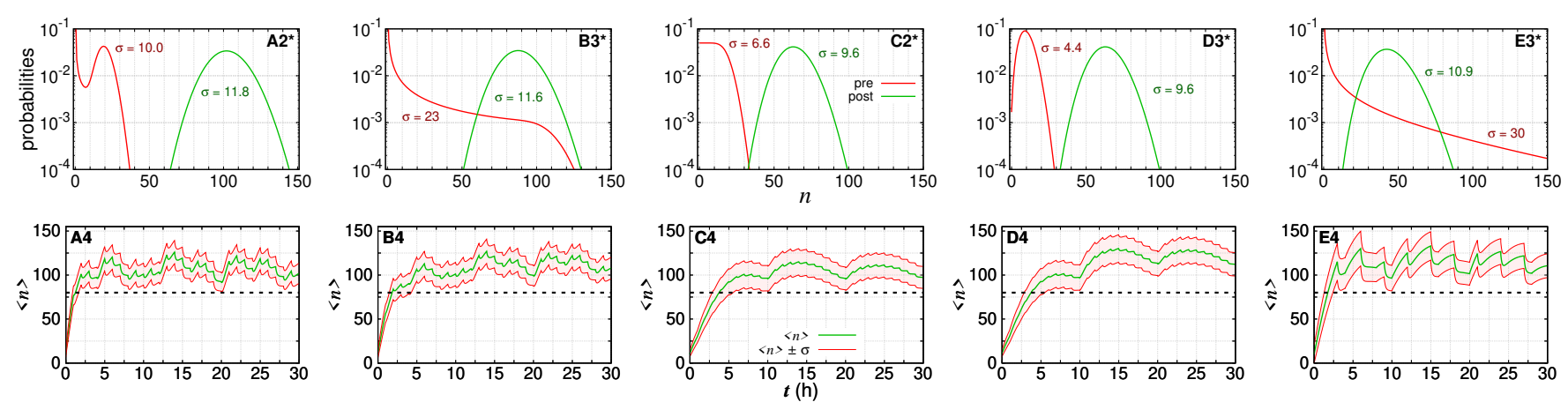

Figure 6. The enhanced treatment designs resulting from distributions of Graphs A2, B3, C2, D3 and E3 of Fig. 2. We simulate a hypothetical cocktail of drugs changing all rates $(f, k, h, \rho)$. The first row of graphs shows the pre (red lines) and post (green lines) treatment probability distributions governing the mRNA numbers. An asterisk is added to the labels to indicate that we are simulating the enhanced treatment design. The average number $\langle n\rangle$ for all pre-treatment distributions is 10 and for each post-treatment distribution is $(103,88,63,63,44)$ following graphs from left to right, respectively. The second row shows the respective dynamic of responses to enhanced treatment design in Graphs A4-E4. Green lines indicate the average numbers of RKIP mRNAs, $\langle n\rangle$. Red lines shows the one standard deviation around the average, $\langle n\rangle \pm \sigma$. Black dashed lines at $\langle n\rangle=80$ indicate the threshold separating the mRNA expression levels in a healthy (above line) and cancer (below) cell. The parameters of post-treatment distributions and the agenda of doses of each enhanced treatment design are shown in Appendix B.

To enhance the previously ineffective treatments designs, we used the aforementioned optimization approach. Drugs dose and interval of application are set to enable $\epsilon_{1}=91$, which was found to be the value leading to the successful treatment design shown in Graphs D1 of Fig. 2. Indeed, that resulted on the best response dynamics of average number of RKIP mRNA's as shown in Graph I1 of Fig. 3.

Graphs A4 and B4 show the dynamics of response to enhanced treatment when pretreatment RKIP gene transcription is governed by a slow switching promoter giving raise to bimodal probability distributions $\left(\epsilon_{0} \leq 1\right)$. The previously ineffective treatment design aimed a bimodal and table-shaped regimens are, respectively, shown by green lines of Graphs A2 and B3 in Fig. 2. The dynamics of response to the enhanced treatment designs are shown in Graphs A2* and B3* of Fig. 6. Both optimized responses resulted from similar changes in kinetic parameters that caused an increase on $A_{1}$ and a reduction on $N_{1}$ and have similar agendas (detailed in Appendix B).

Enhanced treatment design aiming a pre-treatment fast switching gene shown in Graphs C4 and D4 of Fig. 6. The initial probability distributions are table-shaped and quasi-poissonian and have $\epsilon_{0}>1$. Previously, the response dynamics to ineffective treatment designs are shown in Graphs $\mathbf{C} 2$ and D3 of Fig. 2. The response to enhanced treatment having reduced heterogeneity and increased speed is shown in Graphs C2* and D3* of Fig. 6). The enhanced treatment agendas were the same in both cases (see parameters and agendas in Appendix B). The value of $\langle n\rangle_{1}$ was reduced in comparison to the ineffective treatment design because of the decrease of $N_{1}$ not being compensated by the increase of $A_{1}$.

The ineffective treatment design for an initially bursty regimen of RKIP transcription shown in Graph E3 of Fig. 2) is enhanced. The resulting post-treatment distribution is a quasi-poissonian. The dynamics of response to enhanced treatment design is shown, respectively, in Graphs E3* and E4 of Fig. 6. This enhancement required the largest reductions in $\langle n\rangle_{1}$ in comparison to the ineffective design by increasing $A_{1}$ and decreasing $N_{1}$. Note, however, the clear reduction on the standard deviations (heterogeneity) of the response. The parameters values and agenda applied are detailed in Appendix B. 


\section{Discussion}

When the binary model for regulation of gene expression is considered, it is useful to characterize the pre-treatment gene expression regime in terms of $A_{0}, \epsilon_{0}$, and $N_{0}$ (see Eq. 12). Those rates will affect the pre-existing (and post-treatment) noise on the number of mRNAs, see Eqs. 13 and 14 (and Figs. 3, 4, 5 and 6). Furthermore, the time of response to treatment also depends on $\epsilon_{0}$, because the decaying to steady state regime of the average number of mRNAs and its variance have both $\rho$ and $\epsilon \rho$ as their smallest rates (see Eq. 15). For $\epsilon>1$ and $<1$ the time for the system to reach the steady state is, respectively, $\propto \rho^{-1}$ and $\propto(\epsilon \rho)^{-1}$.

All trajectories of the average values shown in graphs of Fig. 4 reach a maximal (Graphs A2 to E2) or cross the threshold (Graphs F2 to J2) after an approximately fixed interval. That is because the component $\propto \mathrm{e}^{-\epsilon \rho t}$ of $\langle n\rangle(t)$ is null as the treatment do not change the steady state probability for the gene to be ON (see Eq. A4). Additionally, the noise on treatment response, measured in terms of the trajectories of $\langle n\rangle(t) \pm \sigma(t)$, is larger when we have the smaller values of the relative switching speed. Inspection of trajectories shown in graphs A to D and F to I of Figs. 3, 4, and 5 helps to identify those features.

The pre-treatment condition which enables the fastest and least heterogeneous response takes place for higher values of the relative switching speed and for the probability for the gene to be ON being $>0.1$. Graphs D and I of Figs. 3, 4, and 5 show that those are the conditions enabling the smallest differences between trajectories $\langle n\rangle(t) \pm \sigma(t)$. At this limit the gene is behaving as a quasi-Poissonian source of transcripts [19]. As the value of the pre-treatment switching speed is reduced from towards two, we have an increase on the noise of the treatment response, though the speed of response does not increase significantly, as shown in graphs $\mathbf{C}$ and $\mathbf{H}$ of Figs. 3, 4, and 5.

The bursty pre-treatment regime $\left(\epsilon_{0}=10\right)$ leads to the noisiest responses to treatment, although being among the fastest ones. The noisy response to treatment occurs partially because when we simulated treatment scenarios shown in graphs $\mathbf{E}$ and $\mathbf{J}$ of Figs. 3, 4, and 5 , the value of the $A(t)$ remained small. In that case, the dynamics would still remain in a transcriptional bursty regime. The small increase on $A(t)$ is because we assumed that the maximal tolerated dose of the drug responsible for increasing $f$ would cause the probability of the ON state to be $10 \times$ larger, independent of its pre-treatment value. Such an assumption needs to be confirmed by experimental studies and an alternative formulation might be proposed because of a lack of confirmation. Graphs B to $\mathbf{E}$ and $\mathbf{G}$ to $\mathbf{J}$ of Figs. 3, 4, and 5 show that the time of response to treatment are all similar since the rates of decay to steady state are $\leq \rho$ for $\epsilon \leq 1$.

The slow relative switching speed pre-treatment condition (here we set $\epsilon_{0}=0.1,1$ ) establishes a challenging regime to be approached. The response is slower and noisier if one takes the strategies considered here. Graphs $\mathbf{A}$ and $\mathbf{F}$ of Figs. 3 and 5 indicate that the increase on the average number of mRNA is the slowest when we have the minimal $\epsilon$ despite treatment increasing $f$ will cause an increase of the relative switching speed.

In the high noise responses the trajectories $\langle n\rangle(t)-\sigma(t)$, do not cross the threshold. It indicates that, in a population of cells having the same regulatory conditions of expression of RKIP gene, the response to treatment is heterogeneous and may be insufficient. Therefore, the dosage and target need to be constructed to ensure a reduction on the response heterogeneity. That can be carried out by increasing the switching speed such that the gene expression will approach a quasi-Poissonian regime. Increasing the switching speed has also the benefit of reducing the time of response to therapy.

The responses to enhanced treatments shown in Fig. 6 (Graphs A4-E4) indicate the potential of the our approach to help on the design of low dose multi-drugs administered in pulses. The three qualitatively different pre-treatment regimens, namely slow bimodal, fast quasi-poissonian, and bursty, have distinguishable response curves. Those are resulting from specificities of the enhanced treatment design aiming at keeping average mRNA numbers and deviations (heterogeneity) above the threshold. 
The synthesis of RKIP protein may have a non-linear dependence of the mRNA numbers, because translation can be regulated by mechanisms such as mRNA stability, translational control and proteosomal degradation [50]. Hence, a theoretical approach considering translation might required the addition of two or more characteristic timescales depending on protein synthesis and degradation [53]. We emphasize that the amounts of RKIP mRNAs in our model are the net effect of the enhanced treatment, since its expression results from the interaction with a complex gene network. Our approach, however, provides the building blocks of gene networks [15] that can be used to understand the functioning of larger modules [10-12] and, hence, to further enhance treatment designs of cancer at metastatic stage.

To further improve our approach one needs to perform a data-based validation of our model. One possible experiment would involve the decrease of RKIP levels, using a siRNA approach to simulate different levels of activation of the RKIP gene. This would be a more specific and precise way to interfere in the RKIP pathway than using 5-AzaC. We could make different levels of RKIP inhibition by siRNA and see if the modeling can predict MEK and ERK phosphorylation levels, the expression of MAPK-induced genes, and the consequence of these activations in cell migration, proliferation, and invasion. The siRNA approach is not able to check the OFF state of the RKIP gene, however it is possible to use a CRISPR/CAS9 system to eliminate the RKIP gene, and then have the OFF state information. Inference methods would be employed on the estimation of parameter values and RKIP mRNA numbers.

Those experiments would help on the understanding of how the environmental information is processed by cells by investigating how regulation of expression of RKIP gene affects its relative downstream kinases. In a recent work, we demonstrated that the slow switching speed regime maximizes the mutual information between the number of transcripts and promoter activity [28]. This regime also coincides with condition of highly heterogeneous and slow response to treatment. Hence, we may ask whether it is relevant for a master regulatory gene to transmit information about its own promoter state and in which cellular context, namely, in a cancerous or healthy one. The answer to this question is important because it helps us to understand the role of noise and the conditions under which its reduction is desirable [27] as it happens when we have a negatively self-regulating gene.

\section{Conclusions}

In this manuscript we present a stochastic binary model for transcription of RKIP gene having treatment induced time-dependent kinetic rates. The exact solutions of this model are approximated using the exact solutions of the equivalent model having stationary kinetic rates. This is the simplest exactly solvable model to describe the regulated transcription of RKIP gene. That enable us to simulate the effect of the application of a drug that changes one (or multiple) kinetic parameters participating on the regulation of RKIP gene. To demonstrate the usefulness of our approach, we simulate three scenarios in which we aim to increase the number of RKIP mRNAs by means of increasing the: $i$. OFF to ON switching rate using DETANONOate; $i$. the synthesis rate using 5-AzaC; iii. both rates of a gene using both drugs together. We show that treatment response speed and heterogeneity depends on the pre-treatment state of the gene. Then, we present an enhanced treatment design that ensures reduced heterogeneity and time of response. Besides useful to inspect treatment designs, the response to treatment may be used on inference of the kinetic constants of a given gene in a synthetic system.

Author Contributions: Conceptualization, AFR; methodology, AFR; software, GG, LRG and AFR; validation, GG, LRCB, LRG, TCTJ and AFR; formal analysis, GG, LRCB, LRG, TCTJ and AFR; investigation, GG and AFR; resources, AFR; data curation, GG, LRG and AFR; writing-original draft preparation, AFR; writing — review and editing, GG, LRCB, LRG, TCTJ and AFR; visualization, GG and AFR; supervision, AFR; project administration, AFR; funding acquisition, AFR. 
Funding: The authors thank funders for support: GG thanks CAPES and PPG-Modelagem de Sistemas Complexos (88887.506433/2020-00); LRCB thanks PRONON Retratos da Mama (25000.069252/201579); TCTJ thanks FAPESP (2015/22814-5); LRG thanks Programa Unificado de Bolsas (PUB-USP) and Pró-Reitoria de Graduação da USP; and AFR thanks CAPES (88881.062174/2014-01) and FAPESP (2012/24962-3).

Acknowledgments: We would like to thank Prof. Marsha R. Rosner and Prof. Roger Chammas for stimulating and helpful discussions that contributed for the concept behind this story.

Conflicts of Interest: The authors declare no conflict of interest.

\section{Appendix A. Formulae}

Appendix A.1. Steady state quantities

The steady state values of the moments of the stochastic binary model for regulation of gene expression are written explicitly. Those quantities were computed elsewhere $[15,17,54]$ and here we denote the steady state probabilities of finding $n$ gene products and the gene ON or OFF as $\tilde{\alpha}_{n}$ or $\tilde{\beta}_{n}$, respectively. The steady state probability of finding $n$ gene products is denoted by $\tilde{\phi}_{n}$, where $\tilde{\phi}_{n}=\tilde{\alpha}_{n}+\tilde{\beta}_{n}$, and explicitly, it is given by:

$$
\tilde{\phi}_{n}\left(A_{s}, \epsilon, N\right)=\frac{N^{n}}{n !} \frac{\left(A_{s} \epsilon\right)_{n}}{(\epsilon)_{n}} \mathrm{M}\left(A_{s} \epsilon+n, \epsilon+n,-N\right)
$$

where $(a)_{n} \equiv a(a+1) \cdots(a+n-1)$ is the Pochhammer symbol and $\mathrm{M}(a, b, z)=\sum_{m=0}^{\infty} \frac{(a)_{m}}{(b)_{m}} \frac{z^{m}}{m !}$ is the KummerM function ([55], page 503). The probability of finding the gene ON, denoted by $A_{s}$, is $A_{s}=\sum_{n=0}^{\infty} \tilde{\alpha}_{n}$. The average number of gene products, denoted by $\langle n\rangle_{s}$, is $\langle n\rangle_{s}=\sum_{n=0}^{\infty} n \tilde{\phi}_{n}$. The partial average number of gene products for the gene being ON, denoted by $\left\langle n_{\alpha}\right\rangle_{s}$, is $\left\langle n_{\alpha}\right\rangle_{s}=\sum_{n=0}^{\infty} n \tilde{\alpha}_{n}$. The average square number of gene products, denoted by $\left\langle n^{2}\right\rangle_{s}$, is $\left\langle n^{2}\right\rangle_{s}=\sum_{n=0}^{\infty} n^{2} \tilde{\phi}_{n}$. Eq. (12) gives the definition of $A_{s}$ as a function of the kinetic constants. The remaining moments can be written in terms of the parameters given at Eq. (12),

$$
\begin{aligned}
\left\langle n_{\alpha}\right\rangle_{s} & =A_{s} N \frac{1+A_{s} \epsilon}{1+\epsilon} \\
\left\langle n^{2}\right\rangle_{s} & =A_{s} N\left(1+N \frac{1+A_{s} \epsilon}{1+\epsilon}\right) .
\end{aligned}
$$

Appendix A.2. Coefficients of Eqs. (15) and (16)

278

$$
\begin{gathered}
Y=N \frac{\left(A_{0}-A_{s}\right)}{1-\epsilon} \\
V=\langle n\rangle_{0}-Y-\langle n\rangle_{s}, \\
U_{1}=U-2 Y\langle n\rangle_{s}, \quad W_{1}=W-2 Y V, \quad X_{1}=X-V^{2},
\end{gathered}
$$

$$
\begin{aligned}
U & =\Upsilon\left[1+2 N \frac{1-\epsilon\left(1-A_{s}\right)}{2-\epsilon}\right], \\
W & =\frac{2 N}{1-\epsilon}\left[N\left(1-A_{s}\right) \frac{\epsilon\left(A_{s}-A_{0}\right)-A_{0}}{\epsilon+1}-A_{s}\langle n\rangle_{0}+\left\langle n_{\alpha}\right\rangle_{0}\right], \\
X & =\left\langle n^{2}\right\rangle_{0}-\left\langle n^{2}\right\rangle_{s}-U-\left(1+2\langle n\rangle_{s}\right) V-W .
\end{aligned}
$$

Appendix A.3. Exact formulas for $A(t),\left\langle n_{\alpha}\right\rangle(t)$, and $\left\langle n^{2}\right\rangle(t)$

$$
A(t)=A_{s}+\left(A_{0}-A_{s}\right) e^{-(h+f) t},
$$




$$
\begin{gathered}
\left\langle n_{\alpha}\right\rangle(t)=\left\langle n_{\alpha}\right\rangle_{s}+R e^{-(h+f) t}+S e^{-\rho t}+T e^{-(f+h+\rho) t}, \\
\left\langle n^{2}\right\rangle(t)=\left\langle n^{2}\right\rangle_{s}+U e^{-(h+f) t}+\left(1+2\langle n\rangle_{s}\right) V e^{-\rho t}+W e^{-(f+h+\rho) t}+X e^{-2 \rho t},
\end{gathered}
$$

where

$$
\begin{aligned}
R & =Y\left[1-\epsilon\left(1-A_{s}\right)\right], \\
S & =A_{s}\langle n\rangle_{0}+\langle n\rangle_{s} \frac{A_{s} \epsilon-A_{0}}{1-\epsilon}, \\
T & =\left\langle n_{\alpha}\right\rangle_{0}-\left\langle n_{\alpha}\right\rangle_{s}-R-S .
\end{aligned}
$$

\section{Appendix B. Treatment parameters and the agenda of doses for enhanced treatment designs}

The following table shows the treatment parameters used to obtain the graphs of Fig. 6. The post-treatment kinetic rates, $\left(k_{1}, \rho_{1}, f_{1}, h_{1}\right)$, and distributions parameters, $\left(N_{1}, A_{1},\langle n\rangle_{1}, \sigma_{1}\right)$, where set to enable the enhanced treatment designs. The number of doses is represented by $n_{i}$ and the time interval between them by $\tau_{i}$, where $i$ indicates the aimed kinetic rate. For all treatments, $\epsilon_{1}=91, n_{f}=3$ and $\tau_{f}=10$.

\begin{tabular}{ccccc}
\hline Graph & $\left(k_{1}, \rho_{1}, f_{1}, h_{1}\right)$ & $\left(N_{1}, A_{1},\langle n\rangle_{1}, \sigma_{1}\right)$ & $\left(n_{k}, n_{\rho}, n_{h}\right)$ & $\left(\tau_{k}, \tau_{\rho}, \tau_{h}\right)$ \\
\hline A4 & $(112,0.83,58,18)$ & $(135,0.76,103,11.8)$ & $(8,30,30)$ & $(4,1,1)$ \\
B4 & $(91,0.67,40,21)$ & $(136,0.65,88,11.6)$ & $(8,30,15)$ & $(4,1,2)$ \\
C4/D4 & $(44,0.42,23,15)$ & $(106,0.6,63.6,9.6)$ & $(30,30,30)$ & $(1,1,1)$ \\
E4 & $(235,1.17,23,83)$ & $(201,0.22,44,10.9)$ & $(8,10,10)$ & $(4,3,3)$ \\
\hline
\end{tabular}

\section{References}

1. Yesilkanal, A.E.; Yang, D.; Valdespino, A.; Tiwari, P.; Sabino, A.U.; Nguyen, L.C.; Lee, J.; Xie, X.H.; Sun, S.; Dann, C.; RobinsonMailman, L.; Steinberg, E.; Stuhlmiller, T.; Frankenberger, C.; Goldsmith, E.; Johnson, G.L.; Ramos, A.F.; Rosner, M.R. Limited inhibition of multiple nodes in a driver network blocks metastasis. eLife 2021, 10. doi:10.7554/elife.59696.

2. Yesilkanal, A.E.; Johnson, G.L.; Ramos, A.F.; Rosner, M.R. New strategies for targeting kinase networks in cancer. J. Biol. Chem. 2021, p. 101128. doi:10.1016/j.jbc.2021.101128.

3. Marusyk, A.; Almendro, V.; Polyak, K. Intra-tumour heterogeneity: a looking glass for cancer? Nat. Rev. Cancer 2012, 12, 323-334. doi:10.1038/nrc3261.

4. Michor, F.; Beal, K. Improving Cancer Treatment via Mathematical Modeling: Surmounting the Challenges Is Worth the Effort. Cell 2015, 163, 1059-1063. doi:10.1016/j.cell.2015.11.002.

5. Alizadeh, A.A.; Aranda, V.; Bardelli, A.; Blanpain, C.; Bock, C.; Borowski, C.; Caldas, C.; Califano, A.; Doherty, M.; Elsner, M.; Esteller, M.; Fitzgerald, R.; Korbel, J.O.; Lichter, P.; Mason, C.E.; Navin, N.; Pe'er, D.; Polyak, K.; Roberts, C.W.M.; Siu, L.; Snyder, A.; Stower, H.; Swanton, C.; Verhaak, R.G.W.; Zenklusen, J.C.; Zuber, J.; Zucman-Rossi, J. Toward understanding and exploiting tumor heterogeneity. Nat. Med. 2015, 21, 846-853. doi:10.1038/nm.3915.

6. Brock, A.; Krause, S.; Ingber, D.E. Control of cancer formation by intrinsic genetic noise and microenvironmental cues. Nat. Rev. Cancer 2015, 15, 499-509. doi:10.1038/nrc3959.

7. Welch, D.R. Tumor Heterogeneity - A ‘Contemporary Concept' Founded on Historical Insights and Predictions. Cancer Res. 2016, 76, 4-6. doi:10.1158/0008-5472.can-15-3024.

8. Guinn, M.T.; Wan, Y.; Levovitz, S.; Yang, D.; Rosner, M.R.; Balázsi, G. Observation and Control of Gene Expression Noise: Barrier Crossing Analogies Between Drug Resistance and Metastasis. Front. Genet. 2020, 11. doi:10.3389/fgene.2020.586726.

9. Bhalla, U.S.; Iyengar, R. Emergent Properties of Networks of Biological Signaling Pathways. Science 1999, $283,381-387$. doi:10.1126/science.283.5400.381.

10. Balázsi, G.; van Oudenaarden, A.; Collins, J.J. Cellular decision making and biological noise: from microbes to mammals. Cell 2011, 144, 910-925. doi:10.1016/j.cell.2011.01.030.

11. Ferrell, J.E. Perfect and Near-Perfect Adaptation in Cell Signaling. Cell Systems 2016, 2, 62-67. doi:10.1016/j.cels.2016.02.006.

12. Gérard, C.; Gonze, D.; Goldbeter, A. Revisiting a skeleton model for the mammalian cell cycle: From bistability to Cdk oscillations and cellular heterogeneity. J. Theor. Biol. 2019, 461, 276-290. doi:10.1016/j.jtbi.2018.10.042.

13. Purvis, J.E.; Lahav, G. Encoding and Decoding Cellular Information through Signaling Dynamics. Cell 2013, 152, 945-956. doi:10.1016/j.cell.2013.02.005.

14. Levine, J.H.; Lin, Y.; Elowitz, M.B. Functional Roles of Pulsing in Genetic Circuits. Science 2013, 342, 1193-1200. doi:10.1126/science.1239999. 
15. Ramos, A.F.; Innocentini, G.C.P.; Forger, F.M.; Hornos, J.E.M. Symmetry in biology: from genetic code to stochastic gene regulation. IET Syst. Biol. 2010, 4, 311-329. doi:10.1049/iet-syb.2010.0058.

16. Peccoud, J.; Ycart, B. Markovian modelling of gene product synthesis. Theor. Popul. Biol. 1995, 48, 222-234. doi:10.1006/tpbi.1995.1027.

17. Iyer-Biswas, S.; Hayot, F.; Jayaprakash, C. Stochasticity of gene products from transcriptional pulsing. Phys. Rev. E 2009, 79, 031911. doi:10.1103/PhysRevE.79.031911.

18. Ramos, A.F.; Gama, L.R.; Morais, M.C.C.; Martins, P.C.M. Stochastic modeling for investigation of the regulation of transcription of the RKIP gene. In Prognostic and Therapeutic Applications of RKIP in Cancer; Baritaki, B.B.S., Ed.; Elsevier, 2020; pp. 257-276. doi:10.1016/b978-0-12-819612-0.00014-6.

19. Giovanini, G.; Sabino, A.U.; Barros, L.R.C.; Ramos, A.F. A comparative analysis of noise properties of stochastic binary models for a self-repressing and for an externally regulating gene. Math. Biosci. Eng. 2020, 17, 5477-5503. doi:10.3934/mbe.2020295.

20. Bonavida, B.; Baritaki, S., Eds. Prognostic and Therapeutic Applications of RKIP in Cancer; Academic Press, 2020. doi:10.1016/C20190-00062-3.

21. Martinho, O.; Granja, S.; Jaraquemada, T.; Caeiro, C.; Miranda-Gonçalves, V.; Honavar, M.; Costa, P.; Damasceno, M.; Rosner, M.R.; Lopes, J.M.; Reis, R.M. Downregulation of RKIP Is Associated with Poor Outcome and Malignant Progression in Gliomas. PLoS One 2012, 7, e30769. doi:10.1371/journal.pone.0030769.

22. Martinho, O.; Pinto, F.; Granja, S.; Miranda-Gonçalves, V.; Moreira, M.A.R.; Ribeiro, L.F.J.; di Loreto, C.; Rosner, M.R.; LongattoFilho, A.; Reis, R.M. RKIP Inhibition in Cervical Cancer Is Associated with Higher Tumor Aggressive Behavior and Resistance to Cisplatin Therapy. PLoS One 2013, 8, e59104. doi:10.1371/journal.pone.0059104.

23. Lamiman, K.; Keller, J.M.; Mizokami, A.; Zhang, J.; Keller, E.T. Survey of Raf Kinase Inhibitor Protein (RKIP) in Multiple Cancer Types. Crit. Rev. Oncog. 2014, 19, 455-468. doi:10.1615/critrevoncog.2014011987.

24. Yesilkanal, A.; Rosner, M. Targeting Raf Kinase Inhibitory Protein Regulation and Function. Cancers $2018,10,306$. doi:10.3390/cancers10090306.

25. Dangi-Garimella, S.; Yun, J.; Eves, E.M.; Newman, M.; Erkeland, S.J.; Hammond, S.M.; Minn, A.J.; Rosner, M.R. Raf kinase inhibitory protein suppresses a metastasis signalling cascade involving LIN28 and let-7. EMBO J. 2009, 28, 347-358. doi:10.1038/emboj.2008.294.

26. Fu, Z.; Smith, P.C.; Zhang, L.; Rubin, M.A.; Dunn, R.L.; Yao, Z.; Keller, E.T. Effects of Raf Kinase Inhibitor Protein Expression on Suppression of Prostate Cancer Metastasis. J. Natl. Cancer Inst. 2003, 95, 878-889. doi:10.1093/jnci/95.12.878.

27. Ramos, A.F.; Reinitz, J. Physical implications of so $(2,1)$ symmetry in exact solutions for a self-repressing gene. J. Chem. Phys. 2019, 151, 041101. doi:10.1063/1.5105361.

28. Gama, L.R.; Giovanini, G.; Balázsi, G.; Ramos, A.F. Binary Expression Enhances Reliability of Messaging in Gene Networks. Entropy 2020, 22, 479. doi:10.3390/e22040479.

29. Yeung, K.; Seitz, T.; Li, S.; Janosch, P.; McFerran, B.; Kaiser, C.; Fee, F.; Katsanakis, K.D.; Rose, D.W.; Mischak, H.; Sedivy, J.M.; Kolch, W. Suppression of Raf-1 kinase activity and MAP kinase signalling by RKIP. Nature 1999, 401, 173-177. doi:10.1038/43686.

30. Yesilkanal, A.E.; Rosner, M.R. Raf Kinase Inhibitory Protein (RKIP) as a Metastasis Suppressor: Regulation of Signaling Networks in Cancer. Crit. Rev. Oncog. 2014, 19, 447-454. doi:10.1615/critrevoncog.2014012000.

31. Zhao, J.; Wenzel, S. Interactions of RKIP with Inflammatory Signaling Pathways. Crit. Rev. Oncog. 2014, 19, 497-504. doi:10.1615/critrevoncog.2014011950.

32. Datar, I.; Tegegne, H.; Qin, K.; Al-Mulla, F.; Bitar, M.S.; Trumbly, R.J.; Yeung, K.C. Genetic and Epigenetic Control of RKIP Transcription. Crit. Rev. Oncog. 2014, 19, 417-430. doi:10.1615/critrevoncog.2014012025.

33. Galal, Y.; Zaravinos, A.; Bonavida, B. Regulation of NKG2D by RKIP: Implications on NK-mediated cytotoxicity and cytokine production. In Successes and Challenges of NK Immunotherapy; Elsevier, 2021; pp. 233-265. doi:10.1016/b978-0-12-824375-6.00012-6.

34. Wei, H.; Liu, Z.; She, H.; Liu, B.; Gu, J.; Wei, D.; Zhang, X.; Wang, J.; Qi, S.; Ping, F. Promoter methylation and expression of Raf kinase inhibitory protein in esophageal squamous cell carcinoma. Oncol. Lett. 2017, 13, 1866-1872. doi:10.3892/ol.2017.5617.

35. Li, D.X.; Cai, H.Y.; Wang, X.; Feng, Y.L.; Cai, S.W. Promoter methylation of Raf kinase inhibitory protein: A significant prognostic indicator for patients with gastric adenocarcinoma. Exp. Ther. Med. 2014, 8, 844-850. doi:10.3892/etm.2014.1833.

36. Beach, S.; Tang, H.; Park, S.; Dhillon, A.S.; Keller, E.T.; Kolch, W.; Yeung, K.C. Snail is a repressor of RKIP transcription in metastatic prostate cancer cells. Oncogene 2007, 27, 2243-2248. doi:10.1038/sj.onc.1210860.

37. Labbozzetta, M.; Poma, P.; Vivona, N.; Gulino, A.; D'Alessandro, N.; Notarbartolo, M. Epigenetic changes and nuclear factor- $\kappa B$ activation, but not microRNA-224, downregulate Raf-1 kinase inhibitor protein in triple-negative breast cancer SUM 159 cells. Oncol. Lett. 2015, 10, 3807-3815. doi:10.3892/ol.2015.3787.

38. Ren, G.; Baritaki, S.; Marathe, H.; Feng, J.; Park, S.; Beach, S.; Bazeley, P.S.; Beshir, A.B.; Fenteany, G.; Mehra, R.; Daignault, S.; Al-Mulla, F.; Keller, E.; Bonavida, B.; de la Serna, I.; Yeung, K.C. Polycomb Protein EZH2 Regulates Tumor Invasion via the Transcriptional Repression of the Metastasis Suppressor RKIP in Breast and Prostate Cancer. Cancer Res. 2012, 72, 3091-3104. doi:10.1158/0008-5472.can-11-3546.

39. Lee, J.; Lee, J.; Farquhar, K.S.; Yun, J.; Frankenberger, C.A.; Bevilacqua, E.; Yeung, K.; Kim, E.J.; Balázsi, G.; Rosner, M.R. Network of mutually repressive metastasis regulators can promote cell heterogeneity and metastatic transitions. Proc. Natl. Acad. Sci. U.S.A 2014, 111, E364-E373. doi:10.1073/pnas.1304840111.

40. Baritaki, S.; Huerta-Yepez, S.; Sahakyan, A.; Karagiannides, I.; Bakirtzi, K.; Jazirehi, A.; Bonavida, B. Mechanisms of nitric oxide-mediated inhibition of EMT in cancer. Cell Cycle 2010, 9, 4931-4940. doi:10.4161/cc.9.24.14229. 
41. Yun, J.; Frankenberger, C.A.; Kuo, W.L.; Boelens, M.C.; Eves, E.M.; Cheng, N.; Liang, H.; Li, W.H.; Ishwaran, H.; Minn, A.J.; Rosner, M.R. Signalling pathway for RKIP and Let-7 regulates and predicts metastatic breast cancer. EMBO J. 2011, 30, $4500-4514$. doi:10.1038/emboj.2011.312.

42. Guo, W.; Dong, Z.; Lin, X.; Zhang, M.; Kuang, G.; Zhu, T. Decreased Expression and Aberrant Methylation of Raf Kinase Inhibitory Protein Gene in Esophageal Squamous Cell Carcinoma. Cancer Invest. 2012, 30, 703-711. doi:10.3109/07357907.2012.732164.

43. Kim, G.E.; Kim, N.I.; Lee, J.S.; Park, M.H.; Yoon, J.H. Reduced RKIP Expression is Associated With Breast Neoplastic Progression and is Correlated With Poor Outcomes and Aberrant Methylation in Breast Carcinoma. Appl. Immunohistochem. Mol. Morphol. 2017, 25, 467-474. doi:10.1097/pai.0000000000000323.

44. Minoo, P.; Baker, K.; Goswami, R.; , G.C.; Foulkes, W.D.; Ruszkiewicz, A.R.; Barker, M.; Buchanan, D.; Young, J.; Jass, J.R. Extensive DNA methylation in normal colorectal mucosa in hyperplastic polyposis. Gut 2006, 55, 1467-1474. doi:10.1136/gut.2005.082859.

45. Fukumura, D.; Kashiwagi, S.; Jain, R.K. The role of nitric oxide in tumour progression. Nat. Rev. Cancer 2006, 6, 521-534. doi:10.1038/nrc1910.

46. Bonavida, B. RKIP-Mediated Chemo-Immunosensitization of Resistant Cancer Cells via Disruption of the NF- $\mathrm{kB} / \mathrm{Snail} / \mathrm{YY} 1 / \mathrm{RKIP}$ Resistance-Driver Loop. Crit. Rev. Oncog. 2014, 19, 431-445. doi:10.1615/critrevoncog.2014011929.

47. Zaravinos, A.; Bonavida, B.; Chatzaki, E.; Baritaki, S. RKIP: A Key Regulator in Tumor Metastasis Initiation and Resistance to Apoptosis: Therapeutic Targeting and Impact. Cancers 2018, 10, 287. doi:10.3390/cancers10090287.

48. Hampl, V.; Tristani-Firouzi, M.; Hutsell, T.C.; Archer, S.L. Nebulized nitric oxide/nucleophile adduct reduces chronic pulmonary hypertension. Cardiovasc. Res. 1996, 31, 55-62. doi:10.1016/s0008-6363(95)00172-7.

49. Wishart, D.S.; Feunang, Y.D.; Guo, A.C.; Lo, E.J.; Marcu, A.; Grant, J.R.; Sajed, T.; Johnson, D.; Li, C.; Sayeeda, Z.; Assempour, N.; Iynkkaran, I.; Liu, Y.; Maciejewski, A.; Gale, N.; Wilson, A.; Chin, L.; Cummings, R.; Le, D.; Pon, A.; Knox, C.; Wilson, M. DrugBank 5.0: a major update to the DrugBank database for 2018. Nucleic Acids Res. 2017, 46, D1074-D1082. doi:10.1093/nar/gkx1037.

50. Walker, E.J.; Rosenberg, S.A.; Wands, J.R.; Kim, M. Role of Raf Kinase Inhibitor Protein in Hepatocellular Carcinoma. For. Immunopathol. Dis. Therap. 2011, 2, 195-204. doi:10.1615/forumimmundisther.v2.i2.110.

51. Morozova, N.; Zinovyev, A.; Nonne, N.; Pritchard, L.L.; Gorban, A.N.; Harel-Bellan, A. Kinetic signatures of microRNA modes of action. RNA 2012, 18, 1635-1655. doi:10.1261/rna.032284.112.

52. Hou, W.; Shan, Y.; Zheng, J.; Lambrecht, R.W.; Donohue, S.E.; Bonkovsky, H.L. Zinc mesoporphyrin induces rapid and marked degradation of the transcription factor bach1 and up-regulates HO-1. Biochim. Biophys. Acta, Gene Regul. Mech. 2008, 1779, 195-203. doi:10.1016/j.bbagrm.2008.01.006.

53. Shahrezaei, V.; Swain, P.S. Analytical distributions for stochastic gene expression. Proc. Natl. Acad. Sci. U.S.A. 2008, 105, 1725617261. doi:10.1073/pnas.0803850105.

54. Innocentini, G.C.P.; Hornos, J.E.M. Modeling stochastic gene expression under repression. J. Math. Biol. 2007, 55, 413-431. doi:10.1007/s00285-007-0090-x.

55. Abramowitz, M.; Stegun, I.A., Eds. Handbook of Mathematical Functions: With Formulas, Graphs, and Mathematical Tables, 10th ed.; Vol. 55, National Bureau of Standards Applied Mathematics Series, U.S. Department of Commerce, National Bureau of Standards: Washington, DC, USA, 1972. 